\title{
Overexpression of Mutant Galactose Permease (ScGal2_N376F) Effective for Utilization of Glucose/Xylose or Glucose/ Galactose Mixture by Engineered Kluyveromyces marxianus
}

\begin{abstract}
Deok-Ho Kwon ${ }^{1,3 \dagger}$, Saet-Byeol Kim ${ }^{1 \dagger}$, Jae-Bum Park' ${ }^{1}$, and Suk-Jin Ha ${ }^{1,2,3 *}$
'Department of Bioengineering and Technology, Kangwon National University, Chuncheon 24341, Republic of Korea ${ }^{2}$ Institute of Fermentation and Brewing, Kangwon National University, Chuncheon 24341, Republic of Korea ${ }^{3}$ Interdisciplinary Program in Biohealth-Machinery Convergence Engineering, Kangwon National University, Chuncheon 24341, Republic of Korea

Mutant sugar transporter SCGAL2-N376F was overexpressed in Kluyveromyces marxianus for efficient utilization of xylose, which is one of the main components of cellulosic biomass. K. marxianus ScGal2_N376F, the ScGAL2-N376F-overexpressing strain, exhibited $47.04 \mathrm{~g} / \mathrm{l}$ of xylose consumption and $26.55 \mathrm{~g} / \mathrm{l}$ of xylitol production, as compared to the parental strain $(24.68 \mathrm{~g} / \mathrm{l}$ and $7.03 \mathrm{~g} / \mathrm{l}$, respectively) when xylose was used as the sole carbon source. When a mixture of glucose and xylose was used as the carbon source, xylose consumption and xylitol production rates were improved by $195 \%$ and $360 \%$, respectively, by K. marxianus ScGal2_N376F. Moreover, the glucose consumption rate was improved by $27 \%$ as compared to that in the parental strain. Overexpression of both wild-type SCGAL2 and mutant SCGAL2-N376F showed $48 \%$ and $52 \%$ enhanced sugar consumption and ethanol production rates, respectively, when a mixture of glucose and galactose was used as the carbon source, which is the main component of marine biomass. As shown in this study, ScGAL2-N376F overexpression can be applied for the efficient production of biofuels or biochemicals from cellulosic or marine biomass.
\end{abstract}

Keywords: Cellulosic biomass, marine biomass, Kluyveromyces marxianus, xylose-specific transporter, ScGal2_N376F

\section{Introduction}

As an alternative feedstock to fossil fuels, cellulosic biomass has been the focus of recent research because of its various advantages over edible biomass, i.e., abundance and relatively cheap price [1-4]. However, several processes are required to use cellulosic biomass, and various substrates such as glucose and xylose must be produced. Therefore, it is necessary to be able to shorten these processes, and the employed strain should be capable of using various substrates. The strain Kluyveromyces marxianus is currently known to exhibit thermotolerance, making it an industrially robust yeast, because economical production processes, such as simultaneous saccharification and fermentation (SSF) or simultaneous saccharification and co-fermentation (SSCF), prefer high temperatures [5-7]. Above all, K. marxianus can utilize various substrates including xylose [6, 8-11].

When coexisting with glucose as a substrate, xylose is not utilized substantially due to glucose catabolite repression [7, 12-14]. The main reason for this problem is that xylose is competitively transported into the cell via some hexose transporters, which have higher affinity for glucose than xylose, at high concentrations of glucose [7, $14,15]$. In previous studies, the metabolic analysis of strains that were engineered for enhanced xylose fermentation in a glucose-xylose mixed-sugar substrate, revealed that the fermentation process is affected by not only the changes of xylose transport kinetics but also by increased levels of hexose transporter expression [16-19].

Studies on the GXF1, SUT1, AT5G59250 (HP59), HXT7, and GAL2 genes of hexose transporters show potential for xylose utilization $[15,20,21]$. In particular, studies on $H X T 7$ and $G A L 2$ gene mutations using genetic engineering suggest many possibilities for the application of co-fermentation of glucose and xylose $[15,20,21]$. These results mean that one of the rate-limiting steps in xylose metabolism is xylose transport [16]. Consequently, the xylose-specific transporters, not being inhibited by glucose, are a crucial prerequisite for efficient and 
economical fermentation of xylose in the presence of glucose.

The mutant transporter ScGal2_N376F, derived from S. cerevisiae, has the highest preference for xylose [15, 22, 23]. In this study, the xylose-specific transporter gene, ScGAL2_N376F, was allowed to overexpress in the K. marxianus KCTC $17555 \triangle U R A 3$ strain, and the fermentation capabilities of the parental strain, ScGAL2overexpressing strain, and ScGAL2_N376F-overexpressing strain were verified using only xylose, galactose, glucose, or a combination of these sugars as carbon source.

\section{Materials and Methods}

\section{Strains and Plasmids}

S. cerevisiae CEN.PK was used for cloning of galactose permease gene (ScGAL2). Escherichia coli TOP10 (KCTC 22006) was used for ScGAL2 gene cloning and sequencing. K. marxianus KCTC $17555 \triangle U R A 3$ was kindly provided from Seoul National University. An overexpression plasmid, pJSKM316-GPD, was kindly provided from Sungkyunkwan University [24]. K. marxianus 17694-DH1 was obtained through a directed evolutionary approach and random mutagenesis in a previous paper [25].

\section{Cloning of ScGAL2 Gene and Site-Directed Mutagenesis}

The ScGAL2 gene was amplified from genomic DNA of S. cerevisiae CEN.PK using ScGAL2_Fw and ScGAL2_Rv primers (Table 1). This amplified gene was used for T vector cloning, using a TOPcloner TA Core Kit (Enzynomics Inc., Korea) and then transformed into E. coli TOP10. Site-directed mutagenesis was performed for substitution of asparagine (N) at position 376 to phenylalanine (F) using a QuikChange Lightning Site-Directed Mutagenesis Kit (Agilent Inc., USA). The primers (ScGAL2_N376F_Fw and ScGAL2_N376F_Rv) used for the site-directed mutagenesis were designed using primer design software available online (Table 1).

\section{Construction of Expression Cassettes}

Two pJSKM316-GPD vectors were constructed for expression of ScGAL2 and ScGAL2_N376F in a K. marxianus strain. The target DNAs were amplified using ScGAL2_XbaI_Fw and ScGAL2_XmaI_Rv primers with a restriction enzyme site of XbaI and XmaI (Table 1). The PCR products and PJSKM316-GPD vector were digested with the enzymes, and both were ligated. The expression vector consists of ScURA3 selection marker, $G P D$ promoter, and TYC terminator.

Transformation of ScGAL2 Genes into K. marxianus KCTC 17555DURA3 or K. marxianus 17694-DH1

Both ScGAL2 and ScGAL2_N376F expression cassettes were amplified using primers (Table 1) ranging from ScURA3 gene to CYC terminator. Each cassette was transformed into K. marxianus KCTC $17555 \triangle U R A 3$ or K. marxianus 17694-DH1 using an EZ-Yeast Transformation Kit (MP Inc., USA). K. marxianus transformants were selected in synthetic complete (SC drop-out plate including $6.7 \mathrm{~g} / \mathrm{l}$ yeast nitrogen base w/o amino acids) (BD Difco Inc., France), $0.6 \mathrm{~g} / \mathrm{l} \mathrm{CSM-His-Leu-Trp-Ura} \mathrm{(MP} \mathrm{Inc.),} \mathrm{and} 20 \mathrm{~g} / \mathrm{l}$ glucose at $30^{\circ} \mathrm{C}$.

\section{Fermentation Conditions}

Pre-culture was performed in $5 \mathrm{ml}$ of YP (10 g/l Yeast extract, $20 \mathrm{~g} / \mathrm{l}$ Peptone) media including $20 \mathrm{~g} / \mathrm{l}$ glucose $\left(\mathrm{YPD}_{20}\right)$ at $30^{\circ} \mathrm{C}$ and $200 \mathrm{rpm}$. The cells were inoculated into $50 \mathrm{ml}$ of YP media including $80 \mathrm{~g} / \mathrm{l}$ glucose, xylose, or galactose $\left(\mathrm{YPD}_{80}, \mathrm{YPX}_{80}\right.$, or $\left.\mathrm{YPGal}_{80}\right)$ at $30^{\circ} \mathrm{C}$ and $100 \mathrm{rpm}$. High-temperature fermentations were performed in $\mathrm{YPX}_{80}$ at $40^{\circ} \mathrm{C}$ and $100 \mathrm{rpm}$. Co-fermentation experiments were carried out in YP media including $40 \mathrm{~g} / \mathrm{l}$ glucose and $40 \mathrm{~g} / \mathrm{l}$ xylose $\left(\mathrm{YPD}_{40} \mathrm{X}_{40}\right), 40 \mathrm{~g} / \mathrm{l}$ glucose and $40 \mathrm{~g} / \mathrm{l}$ galactose $\left(\mathrm{YPD}_{40} \mathrm{Gal}_{40}\right)$ at $30^{\circ} \mathrm{C}$ and $100 \mathrm{rpm}$.

\section{Analytical Methods}

Cell densities were measured at $600 \mathrm{~nm}$ using a GENESYS ${ }^{\text {ma }} 10 \mathrm{~S}$ UV-visible spectrophotometer (Thermo Inc., USA). Cells were centrifuged and the supernatants were analyzed by using a high-performance liquid chromatography (HPLC) system (Agilent Inc.) with a Rezex ROA-Organic Acid $\mathrm{H}^{+}$column (Phenomenex Inc., USA) to measure the concentrations of glucose, xylose, galactose, xylitol, acetate, and ethanol. The temperatures of the column and refractive index detector (RID) were maintained at $50^{\circ} \mathrm{C}$ and $0.005 \mathrm{~N} \mathrm{of}_{2} \mathrm{SO}_{4}$ solution was used as a mobile phase at a flow rate of $0.6 \mathrm{ml} / \mathrm{min}$.

Table 1. Primers used in this study.

\begin{tabular}{ll}
\hline \multicolumn{1}{c}{ Primer names } & \multicolumn{1}{c}{ Sequence $\left(5^{\prime} \rightarrow 3^{\prime}\right)$} \\
\hline$S c G A L 2 \_\mathrm{Fw}$ & ATGGCAGTTGAGGAGAACAATATGCC \\
ScGAL2_Rv & TTATTCTAGCATGGCCTTGTACCACG \\
ScGAL2-N376F_Fw & TCCATTGTCATTGGTGTAGTCTTCTTTGCCTCCACTTTCTTTAG \\
ScGAL2-N376F_Rv & CTAAAGAAAGTGGAGGCAAAGAAGACTACACCAATGACAATGGA \\
ScGAL2_XbaI_Fw & GCTCTAGAATGGCAGTTGAGGAGAACAATATGCC \\
ScGAL2_XmaI_Rv & TCCCCCCGGGTTATTCTAGCATGGCCTTGTACCACG \\
Fw_scURA3 & CGG CAT CAG AGC AGA TTG TAC TGA GAG TGC \\
Rv_CYCt & CCT CAC TAA AGG GAA CAA AAG \\
\end{tabular}


A

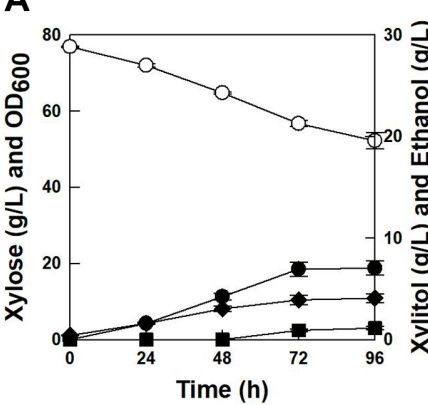

B

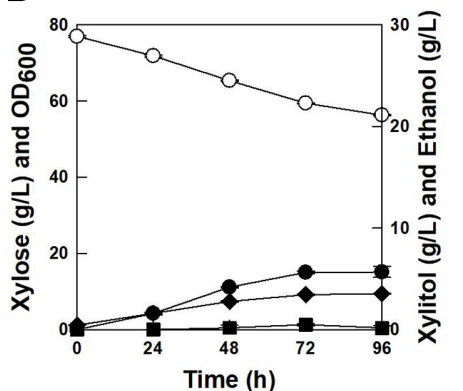

C

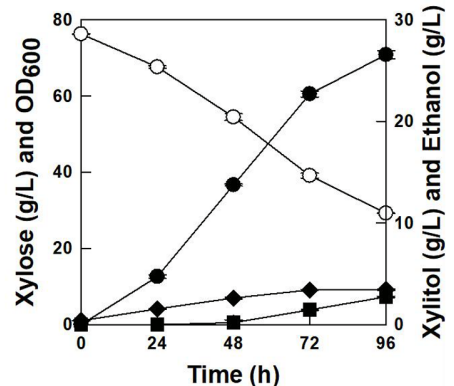

Fig. 1. Time profiles of xylose fermentations by the parental strain (A), K. marxianus ScGal2 (B), and K. marxianus ScGal2_N376F $(\mathrm{C})$ at $30^{\circ} \mathrm{C}$ and $100 \mathrm{rpm}$. Symbols: $x y l o s e ~(\bigcirc)$, OD $(\diamond)$, xylitol $(\boldsymbol{O})$, and ethanol $(\square)$.

\section{Results and Discussion}

Site-Directed Mutagenesis to Engineer a Xylose-Specific Transporter, ScGal2_N376F

Previously, it was reported that ScGal2_N376F mutant had the highest affinity for xylose, along with weakened affinity for glucose, among different hexose transporter mutants from engineered S. cerevisiae [15, 22]. Ogataea polymorpha yeast also exhibited improved xylose utilization during high-temperature alcoholic fermentation by overexpression of engineered $S c G A L 2$ mutants [23]. In this study, ScGAL2 gene was cloned from S. cerevisiae and then ScGAL2_N376F gene was engineered by site-directed mutagenesis. Two adenines at positions 1126 and 1127 of ScGAL2 gene were substituted with thymine, leading to amino acid substitution from asparagine (N) to phenylalanine (F) at position 376. Each expression cassette harboring ScGAL2 or ScGAL2_N376F gene with GPD promoter, TYC terminator, and ScURA3 gene as a selection marker, was introduced into $K$. marxianus $17555 \triangle U R A 3$ to confirm whether wild-type and mutant Gal2 possess different fermentation phenotype.

\section{Improved Xylose Fermentation Capability of Overexpressed ScGAL2_N376F Strain}

Fermentation experiments were performed to compare xylose fermentation capabilities between the parental strain (K. marxianus 17555 ScGal2_N376F) using xylose as a sole carbon source in YPX80 media. For fair comparisons between K. marxianus KCTC $17555 \triangle U R A 3$ and K. marxianus KCTC $17555 \Delta U R A 3+S c U R A 3$, all fermentation results were nearly the same (data not shown). The parental strain consumed $24.68 \mathrm{~g} / \mathrm{l}$ xylose and produced $7.03 \mathrm{~g} / \mathrm{l}$ xylitol with a yield of $0.28 \mathrm{~g} / \mathrm{g}$ for $96 \mathrm{~h}$ (Fig. 1A). The xylose consumption and xylitol production rates were $0.26 \mathrm{~g} / \mathrm{l} / \mathrm{h}$ and $0.07 \mathrm{~g} / \mathrm{l} / \mathrm{h}$, respectively. Likewise, ScGAL2 gene-overexpressing strain, K. marxianus ScGal2, exhibited similar xylose fermentation capability with the parental strain as shown in Fig. 1B. Xylose was consumed at $20.67 \mathrm{~g} / \mathrm{l}$ for $96 \mathrm{~h}$ with a $0.22 \mathrm{~g} / \mathrm{l} / \mathrm{h}$ xylose consumption rate and $5.66 \mathrm{~g} / \mathrm{l}$ xylitol was produced for $96 \mathrm{~h}$ by K. marxianus ScGal2. The xylitol yield and productivity of $K$. marxianus ScGal2 $(0.27 \mathrm{~g} / \mathrm{g}$ and $0.06 \mathrm{~g} / \mathrm{l} / \mathrm{h})$ were almost similar to those from the parental strain. Interestingly, ScGAL2-N376F gene- overexpressing strain, K. marxianus ScGal2_N376F, showed highly enhanced xylose fermentation capability as shown in Fig. 1C. Xylose was consumed at $47.04 \mathrm{~g} / \mathrm{l}$, and $26.55 \mathrm{~g} / \mathrm{l}$ xylitol was produced by $K$. marxianus ScGal2_N376F with the yield of $0.56 \mathrm{~g} / \mathrm{g}$. The xylose consumption rate and xylitol production rate were $0.49 \mathrm{~g} / \mathrm{l} / \mathrm{h}$ and $0.28 \mathrm{~g} / \mathrm{l} / \mathrm{h}$, which were $91 \%$ and $278 \%$ improved, respectively, compared with those from the parental strain. In addition, the xylitol yield improved from $0.28 \mathrm{~g} / \mathrm{g}$ to $0.56 \mathrm{~g} / \mathrm{g}$, which was a 2-fold improvement by $K$. marxianus ScGal2_N376F. These results suggest that substitution of asparagine $(\mathrm{N})$ with phenylalanine $(\mathrm{F})$ at position 376 of $\mathrm{ScG}$ al2 appreciably influences the xylose fermentation capability. Since XylE, xylose $/ \mathrm{H}^{+}$symporter of $E$. coli, showed good accordance with $\mathrm{ScGal} 2$ at transmembrane helices 5 (T219) and 8 (N376), changes at position 376 of ScGal2 could result in alteration of the binding pocket, and the N376F mutation would drastically reduce the space in the central cavity, which may offer an explanation for the xylose specificity of this mutant [15].

\section{Effects of ScGAL2 Gene Overexpression on Galactose or Glucose Utilization}

The parental strain and engineered strains fermented galactose or glucose as a sole carbon source in $\mathrm{YPGal}_{80}$ or $\mathrm{YPD}_{80}$ media, respectively, to verify the effect of $S c G A L 2$ gene overexpression on galactose or glucose utilization. When galactose was used as a sole carbon source, the parental strain consumed $80 \mathrm{~g} / \mathrm{l}$ galactose within $24 \mathrm{~h}$ with a $3.45 \mathrm{~g} / \mathrm{l} / \mathrm{h}$ galactose consumption rate and a $1.62 \mathrm{~g} / \mathrm{l} / \mathrm{h}$ ethanol production rate (Fig. $2 \mathrm{~A}$ ). The two engineered strains K. marxianus ScGal2 and K. marxianus ScGal2_N376F exhibited much higher galactose consumption rates $(5.90 \mathrm{~g} / \mathrm{l} / \mathrm{h}$ and $5.86 \mathrm{~g} / \mathrm{l} / \mathrm{h})$ and ethanol production rates $(2.79 \mathrm{~g} / \mathrm{l} / \mathrm{h}$ and $2.73 \mathrm{~g} / \mathrm{l} / \mathrm{h})$, respectively, than those from the parental strain, because the two engineered strains consumed $80 \mathrm{~g} / \mathrm{l}$ galactose much faster (within $14 \mathrm{~h}$ ). Therefore, galactose consumption rates and ethanol production rates were $69 \sim 71 \%$ and $68 \sim 72 \%$ improved, respectively, by $S c G A L 2$ or $S c G A L 2-N 376 \mathrm{~F}$ gene overexpression. However, the ethanol yields from galactose were similar to the $0.47 \mathrm{~g} / \mathrm{g}$ of the parental strain and the two engineered strains. These results indicated that $S c G A L 2$ or ScGAL2-N376F gene overexpression enhanced the overall galactose consumption rate to a comparable level without a change in ethanol yield. 
A

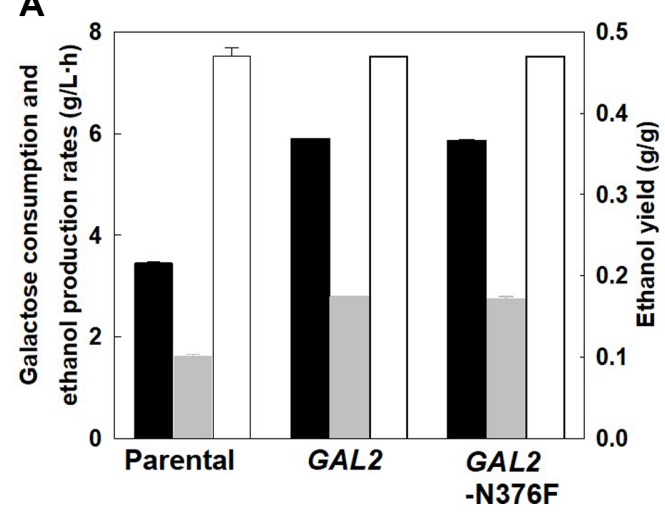

B

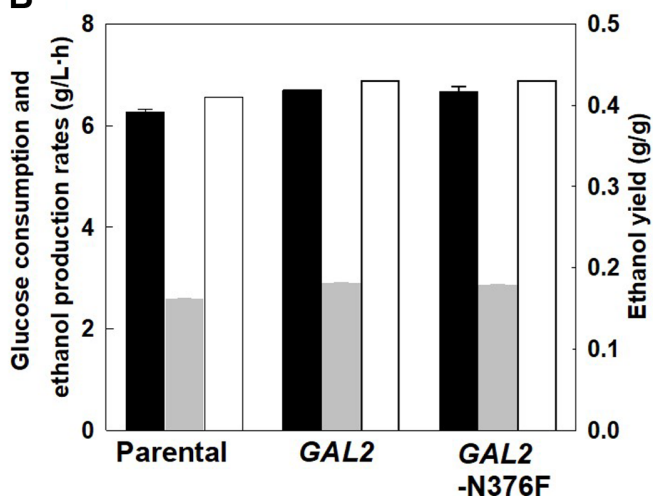

Fig. 2. Comparisons of galactose (A) or glucose (B) fermentation capability by the parental strain, K. marxianus ScGal2, and K. marxianus ScGal2_N376F. Symbols: galactose consumption rate ( $\square$ ), glucose consumption rate ( $\square$ ), ethanol production rate ( $\square$ ), and ethanol yield ( $\square$ ).

When glucose was used as a sole carbon source, glucose consumption rates ( 6.69 and $6.66 \mathrm{~g} / \mathrm{l} / \mathrm{h})$, ethanol production rates $(2.88$ and $2.85 \mathrm{~g} / \mathrm{l} / \mathrm{h})$, and ethanol yields $(0.43$ and $0.43 \mathrm{~g} / \mathrm{g})$ were all slightly improved, respectively, by the two engineered strains as compared to those from the parental strain $(6.27 \mathrm{~g} / \mathrm{l} / \mathrm{h}, 2.59 \mathrm{~g} / \mathrm{l} / \mathrm{h}$, $0.41 \mathrm{~g} / \mathrm{g}$ ) as shown in Fig. 2B. These results indicated that overexpression of $S c G A L 2$ or ScGAL2-N376F gene, even slightly, improved the glucose consumption rate and ethanol yield by the two engineered strains.

The overexpression of the mutant ScGAL2-N376F gene resulted in improved xylose consumption rate, xylitol production rate, and xylitol yield as compared to those from the wild-type $S c G A L 2$ gene overexpression. However, the sugar consumption rate and ethanol production rate were not highly changed when galactose or glucose was used as a sole carbon source, suggesting that the N376F mutation of ScGal2 might not change the hexose sugar (glucose or galactose)-transporting capability, but might improve the pentose sugar (xylose)-transporting capability of the two engineered K. marxianus strains.

Enhanced Mixed Sugar Utilization by ScGAL2_N376F-Overexpressing Strain

As yeasts display strong glucose catabolite repression when glucose and other sugars are used together, sequential utilization of a sugar mixture is a common phenomenon. Therefore, the rate of second sugar utilization highly affects overall productivity after glucose depletion. In this study, fermentation experiments using a sugar mixture (glucose/xylose or glucose/galactose) were performed to verify the effect of ScGAL2 or ScGAL2-N376F overexpression for mixed-sugar fermentation.

When a mixture of $40 \mathrm{~g} / \mathrm{l}$ glucose and $40 \mathrm{~g} / \mathrm{l}$ xylose was used as carbon source, the parental strain exhibited typical sequential utilization of the sugar mixture, which is characterized by fast consumption of glucose at first and very slow consumption of xylose later. K. marxianus ScGal2 and K. marxianus ScGal2_N376F also exhibited sequential utilization of glucose and xylose; however, the rates of utilization for each sugar were different. As shown in Fig. 3, the parental strain consumed $40 \mathrm{~g} / \mathrm{l}$ glucose for $10 \mathrm{~h}$ and then slowly consumed $5.37 \mathrm{~g} / \mathrm{l}$ xylose with $2.51 \mathrm{~g} / \mathrm{l}$ of xylitol production for $24 \mathrm{~h}$. K. marxianus ScGal2 exhibited almost similar fermentation results as the parental strain, however, only glucose consumption rate was higher than that in the parental strain. K. marxianus ScGal2_N376F exhibited higher glucose and xylose consumption rates than those in the parental strain. Through

A

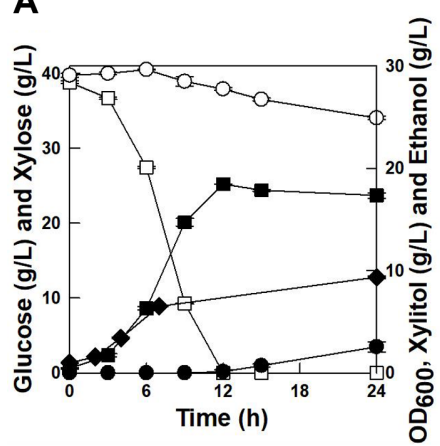

B

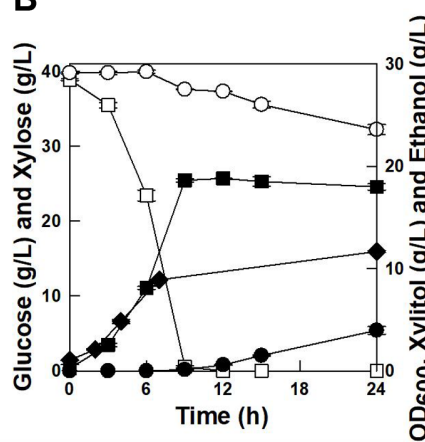

C

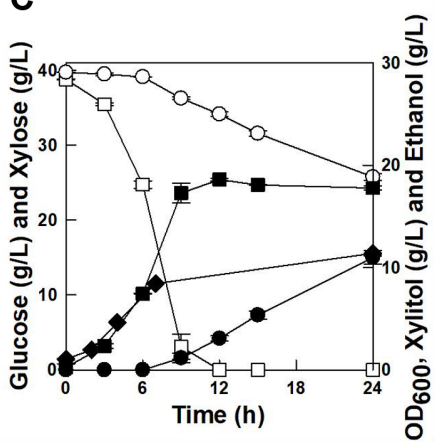

Fig. 3. Comparisons of fermentation results in glucose-xylose mixture by the parental strain (A), K. marxianus ScGal2 (B), and K. marxianus ScGal2_N376F (C). Symbols: glucose ( $\square$ ), xylose ( $\bigcirc)$, xylitol ( $)$, $\mathrm{OD}(\diamond)$, and ethanol $(\boldsymbol{\square})$. 
Table 2. Comparisons of fermentation results by engineered $K$. marxianus strains in YP medium containing glucose-galactose mixture.

\begin{tabular}{|c|c|c|c|}
\hline Results of fermentation & Parental strain & K. marxianus ScGal2 & K. marxianus ScGal2_N376F \\
\hline OD & $11.80 \pm 0.07$ & $19.63 \pm 0.25$ & $18.98 \pm 0.60$ \\
\hline Ethanol & $35.39 \pm 0.00$ & $36.63 \pm 0.26$ & $36.14 \pm 0.82$ \\
\hline Glucose consumption rate & $4.00 \pm 0.10$ & $5.00 \pm 0.00$ & $5.00 \pm 0.00$ \\
\hline Galactose consumption rate & $0.98 \pm 0.06$ & $2.86 \pm 0.00$ & $2.82 \pm 0.00$ \\
\hline Ethanol production rate & $1.69 \pm 0.02$ & $2.62 \pm 0.02$ & $2.58 \pm 0.06$ \\
\hline Ethanol yield & $0.44 \pm 0.00$ & $0.46 \pm 0.00$ & $0.45 \pm 0.01$ \\
\hline
\end{tabular}

the overexpression of $S c G A L 2-\mathrm{N} 376 \mathrm{~F}$ into K. marxianus strain, the overall consumption rate of the glucose-xylose mixture, ethanol productivity, and xylitol productivity were improved by $22 \%, 3 \%$, and $340 \%$, respectively. These results suggest that the overexpression of $S c G A L 2-\mathrm{N} 376 \mathrm{~F}$ in the yeast strain could be very suitable for the utilization of cellulosic biomass composed mainly of glucose and xylose. Since the parental strain (K. marxianus 17555 2 URA3) possesses an inefficient xylose metabolic pathway, only K. marxianus ScGal2_N376F showed improved xylose consumption rate and xylitol production rate. If an efficient xylose-fermenting yeast was used as the parental strain, enhanced ethanol production rate might be achieved along with enhanced xylose consumption rate.

When a mixture of $40 \mathrm{~g} / \mathrm{l}$ glucose and $40 \mathrm{~g} / \mathrm{l}$ galactose was used as carbon source, K. marxianus ScGal2 and K. marxianus ScGal2_N376F also exhibited faster glucose and galactose utilization rates that those from the parental strain as shown in Table 2. The parental strain consumed $40 \mathrm{~g} / \mathrm{l}$ glucose for $10 \mathrm{~h}$ and then consumed $40 \mathrm{~g} / \mathrm{l}$ galactose for $20 \mathrm{~h}$ with $35.39 \mathrm{~g} / \mathrm{l}$ ethanol production. Both K. marxianus ScGal2 and K. marxianus ScGal2_N376F consumed $40 \mathrm{~g} / \mathrm{l}$ glucose for $8 \mathrm{~h}$ and then consumed $40 \mathrm{~g} / \mathrm{l}$ galactose for $14 \mathrm{~h}$ with $36.14 \sim 36.63 \mathrm{~g} / \mathrm{l}$ ethanol production. The overexpression of the wild-type ScGAL2 or the mutant-type ScGAL2_N376F into K. marxianus increased both glucose and galactose consumption rates in a glucose-galactose mixture. Therefore, the overall consumption rate of the glucose-galactose mixture, ethanol productivity and ethanol yield, were improved by $48 \%, 52 \%$, and $2.9 \%$, respectively, by the overexpression of $S c G A L 2-\mathrm{N} 376 \mathrm{~F}$ into K. marxianus strain. These results suggest that K. marxianus ScGal2_N376F could be a very suitable yeast strain for the utilization of marine biomass composed mainly of glucose and galactose.

Xylose fermentation capability was highly improved by overexpression of ScGAL2-N376F into K. marxianus 17694-DH1

According to Fig. 3, the effect of $S c G A L 2-N 376$ F gene overexpression for ethanol production from xylose is not clear because the parental strain (K. marxianus KCTC 17555 UURA3) is not an efficient xylose-fermenting strain. Therefore, ScGAL2-N376F gene was overexpressed into K. marxianus 17694-DH1 which showed more efficient ethanol production capability from xylose, to verify the effect of ScGAL2-N376F overexpression on xylose fermentation [25]. The ScGAL2-N376F-overexpressed strain, K. marxianus 17694-DHG1, exhibited a $0.49 \mathrm{~g} / \mathrm{l} / \mathrm{h}$ xylose consumption rate and a $0.09 \mathrm{~g} / \mathrm{l} / \mathrm{h}$ ethanol production rate, representing $151 \%$ and $242 \%$ improved results, respectively, as compared to those from the parental strain, K. marxianus 17694-DH1 (Fig. 4). In addition, ethanol yield was improved from $0.18 \mathrm{~g} / \mathrm{g}$ to $0.30 \mathrm{~g} / \mathrm{g}$ by $K$. marxianus $17694-\mathrm{DHG} 1$. When ScGAL2_N376F was overexpressed with several key enzymes for xylose utilization into K. marxianus, the engineered strain showed coconsumption of glucose and xylose [7, 26]. However, only ScGAL2-N376F-overexpressed strain K. marxianus 17694-DHG1 did not show co-consumption of glucose and xylose which suggested that the overexpression of only ScGAL2-N376F into K. marxianus is not enough for the co-consumption of glucose and xylose.

A

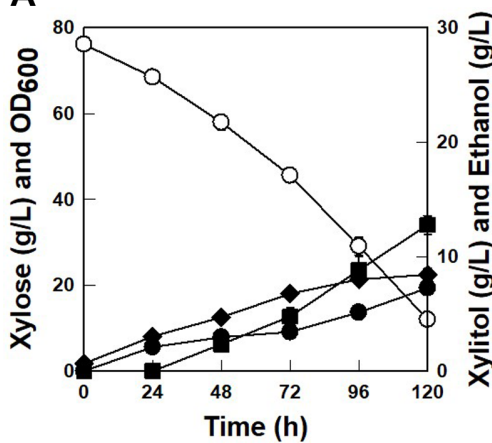

B

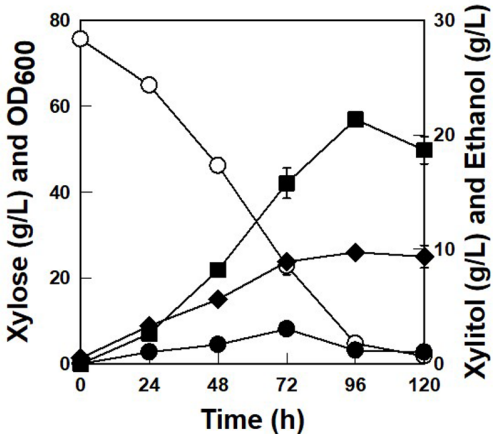

Fig. 4 Time profiles of xylose fermentations by the parental strain (A) and $K$. marxianus 17694-DHG1 (B) at $30^{\circ} \mathrm{C}$ and $200 \mathrm{rpm}$. Symbols: $x y l o s e(\bigcirc), \mathrm{OD}(\bullet), \operatorname{xylitol}(\bullet)$, and ethanol $(\square)$. 


\section{Conclusion}

The efficient utilization of sugar mixtures such as glucose/xylose or glucose/galactose is a prerequisite for the production of fuels and chemicals from cellulosic or marine biomass by engineered yeast. When the mutant sugar transporter ScGAL2-N376F was overexpressed in K. marxianus, xylose utilization rate and xylitol production rate were improved by $195 \%$ and $360 \%$, respectively, with glucose consumption rate improved by $27 \%$ as compared to that in the parental strain, when a glucose/xylose mixture was used as a carbon source. $K$. marxianus ScGal2_N376F also exhibited a 48\% improved overall consumption rate of glucose/galactose mixture and 52\% improved ethanol productivity as compared to that in the parental strain. These results suggest that the ScGAL2$\mathrm{N} 376 \mathrm{~F}-$ overexpressing strain could be very suitable to produce fuels and chemicals from cellulosic or marine biomass.

\section{Acknowledgments}

This research was supported by the Basic Science Research Program through the National Research Foundation of Korea (NRF) funded by the Ministry of Education (2018R1A6A1A03025582 and 2019R1F1A1055315)

\section{Conflict of Interest}

The authors have no financial conflicts of interest to declare.

\section{References}

1. Banerjee S, Mishra G, Roy A. 2019. Metabolic engineering of bacteria for renewable bioethanol production from cellulosic biomass. Biotechnol. Bioprocess Eng. 24:713-733.

2. Lee J. 1997. Biological conversion of lignocellulosic biomass to ethanol. J. Biotechnol. 56: 1-24.

3. Mosier N, Wyman C, Dale B, Elander R, Lee Y, Holtzapple M, et al. 2005. Features of promising technologies for pretreatment of lignocellulosic biomass. Bioresour. Technol. 96: 673-686.

4. Von Sivers M, Zacchi G. 1996. Ethanol from lignocellulosics: a review of the economy. Bioresour. Technol. 56: 131-140.

5. Arora R, Behera S, Sharma NK, Kumar S. 2019. Evaluating the pathway for co-fermentation of glucose and xylose for enhanced bioethanol production using flux balance analysis. Biotechnol. Bioprocess Eng. 24: 924-933.

6. Park J-B, Kim J-S, Jang S-W, Hong E, Ha S-J. 2015. The application of thermotolerant yeast Kluyveromyces marxianus as a potential industrial workhorse for biofuel production. KSBB J. 30: 125-131.

7. Zhang B, Zhang J, Wang D, Han R, Ding R, Gao X, et al. 2016. Simultaneous fermentation of glucose and xylose at elevated temperatures co-produces ethanol and xylitol through overexpression of a xylose-specific transporter in engineered Kluyveromyces marxianus. Bioresour. Technol. 216: 227-237.

8. Anderson P, McNeil K, Watson K. 1986. High-efficiency carbohydrate fermentation to ethanol at temperatures above $40 \mathrm{C}$ by Kluyveromyces marxianus var. marxianus isolated from sugar mills. Appl. Environ. Microbiol. 51: 1314-1320.

9. Fonseca GG, Heinzle E, Wittmann C, Gombert AK. 2008. The yeast Kluyveromyces marxianus and its biotechnological potential. Appl. Microbiol. Biotechnol. 79: 339-354.

10. Kim J-S, Park J-B, Jang S-W, Ha S-J. 2015. Enhanced xylitol production by mutant Kluyveromyces marxianus 36907-FMEL1 due to improved xylose reductase activity. Appl. Biochem. Biotechnol. 176: 1975-1984.

11. Park J-B, Kim J-S, Jang S-W, Kweon D-H, Hong EK, Shin WC, et al. 2016. Sequence analysis of KmXYL1 genes and verification of thermotolerant enzymatic activities of xylose reductase from four Kluyveromyces marxianus strains. Biotechnol. Bioprocess Eng. 21: 581-586.

12. Gancedo JM, Gancedo C. 1986. Catabolite repression mutants of yeast. FEMS Microbiol. Rev. 1: 179-187.

13. Kim SR, Ha S-J, Wei N, Oh EJ, Jin Y-S. 2012. Simultaneous co-fermentation of mixed sugars: a promising strategy for producing cellulosic ethanol. Trends Biotechnol. 30: 274-282.

14. Wang M, Yu C, Zhao H. 2016. Directed evolution of xylose specific transporters to facilitate glucose-xylose co-utilization. Biotechnol. Bioeng. 113: 484-491.

15. Farwick A, Bruder S, Schadeweg V, Oreb M, Boles E. 2014. Engineering of yeast hexose transporters to transport D-xylose without inhibition by D-glucose. Proc. Natl. Acad. Sci. USA 111: 5159-5164.

16. Hector RE, Qureshi N, Hughes SR, Cotta MA. 2008. Expression of a heterologous xylose transporter in a Saccharomyces cerevisiae strain engineered to utilize xylose improves aerobic xylose consumption. Appl. Microbiol. Biotechnol. 80: 675-684.

17. Kuyper M, Toirkens MJ, Diderich JA, Winkler AA, Van Dijken JP, Pronk JT. 2005. Evolutionary engineering of mixed-sugar utilization by a xylose-fermenting Saccharomyces cerevisiae strain. FEMS Yeast Res. 5: 925-934.

18. Saloheimo A, Rauta J, Stasyk V, Sibirny AA, Penttilä M, Ruohonen L. 2007. Xylose transport studies with xylose-utilizing Saccharomyces cerevisiae strains expressing heterologous and homologous permeases. Appl. Microbiol. Biotechnol. 74: 1041-1052.

19. Wahlbom CF, Otero RRC, van Zyl WH, Hahn-Hägerdal B, Jönsson LJ. 2003. Molecular analysis of a Saccharomyces cerevisiae mutant with improved ability to utilize xylose shows enhanced expression of proteins involved in transport, initial xylose metabolism, and the pentose phosphate pathway. Appl. Environ. Microbiol. 69: 740-746.

20. Apel AR, Ouellet M, Szmidt-Middleton H, Keasling JD, Mukhopadhyay A. 2016. Evolved hexose transporter enhances xylose uptake and glucose/xylose co-utilization in Saccharomyces cerevisiae. Sci. Rep. 6: 1-10.

21. Runquist D, Hahn-Hägerdal B, Rådström P. 2010. Comparison of heterologous xylose transporters in recombinant Saccharomyces cerevisiae. Biotechnol. Bbiofuels 3: 5 .

22. Reznicek O, Facey SJ, de Waal PP, Teunissen AW, de Bont JA, Nijland JG, et al. 2015. Improved xylose uptake in Saccharomyces cerevisiae due to directed evolution of galactose permease Gal2 for sugar co-consumption. J. Appl. Microbiol. 119: 99-111.

23. Vasylyshyn R, Kurylenko O, Ruchala J, Shevchuk N, Kuliesiene N, Khroustalyova G, et al. 2020. Engineering of sugar transporters for improvement of xylose utilization during high-temperature alcoholic fermentation in Ogataea polymorpha yeast. Microb. Cell Fact. 19: 96.

24. Lee K-S, Kim J-S, Heo P, Yang T-J, Sung Y-J, Cheon Y, et al. 2013. Characterization of Saccharomyces cerevisiae promoters for heterologous gene expression in Kluyveromyces marxianus. Appl. Microbiol. Biotechnol. 97: 2029-2041.

25. Kwon D-H, Park J-B, Hong E, Ha S-J. 2019. Ethanol production from xylose is highly increased by the Kluyveromyces marxianus mutant 17694-DH1. Bioprocess Biosyst. Eng. 42: 63-70.

26. Zhang B, Zhu Y, Zhang J, Wang D, Sun L, Hong J. 2017. Engineered Kluyveromyces marxianus for pyruvate production at elevated temperature with simultaneous consumption of xylose and glucose. Bioresour. Technol. 224: 553-562. 